REFERENCES:

[1] Mendoza FA, Nagle SJ, Lee JB, Jimenez SA. A prospective observational study of mycophenolate mofetil treatment in progressive diffuse cutaneous systemic sclerosis of recent onset. J Rheumatol. 2012;39:1241-7

[2] Steen VD, Medsger TA Jr. Improvement in skin thickening in systemic sclerosis associated with improved survival. Arthritis Rheum. 2001; 44 (12):2828-35.

Disclosure of Interest: None declared

DOI: 10.1136/annrheumdis-2018-eular.7558

\section{AB0756 MDA 5 DERMATOMYOSISTIS AND RESPONSE TO RITUXIMAB IN A SMALL COHORT AT OUR INSTITUTION}

F. Berianu, V.J. Maya, B. Wang. MAYO CLINIC, Jacksonville, USA

Background: Treatment for anti-melanoma differentiation-associated gene 5 antibody (MDA 5) Dermatomyositis is posing challenging to clinician and there is currently no consensus on therapy. The MDA5 was recently classified as a myositis-specific antibody (MSA), and has been associated with rapidly progressive interstitial lung disease (ILD), amyopathic dermatomyositis (aDM), mechanic's hands, ulcerations, inflammatory arthritis, and increased mortality.

Objectives: This study sought to characterised response to Rituximab in a small cohort of patients with MDA 5 DM at our institution.

Methods: A retrospective chart review was done to identify all MDA5 positive patients who were evaluated at our centre from 2015 to 2017 with suspected myositis and had myositis-associated autoantibodies tested using a commercial pane (MyoMarker Panel 3). For all patients who were positive for MDA5, we collected clinical information on the presence of myopathy, skin involvement and ILD, data on the autoantibody profile, PFTs and high resolution CT pattern along with therapy response.

Results: 11 were positive for MDA5. 7 were females and 4 were males, with mean age 56.6 years. Eight patients were treated with Rituximab from which seven had ILD at presentation: 4 NSIP, 2 with organising pneumonia, one had UIP and one had fatal acute interstitial pneumonitis 2 months after onset of mechanics' hands and inflammatory arthritis. Three patients without ILD were treated with other DMARDs.

All had responded well to Rituximab with improvement in skin ulceration, inflammatory arthritis and mechanics' hands and most patients had stable ILD except for one patient with fulminant interstitial pneumonitis for with Cytoxan was added without any additional benefit.

Conclusions: Rituximab is a good management option for all manifestation of MDA 5 DM. Further studies are needed to elucidate the association of MSA and the clinical manifestations and response to treatment response of patients with inflammatory myopathies.

REFERENCES :

[1] Br J Dermatol. 2017 Feb;176(2):395-402

[2] Am J Pathol. 2016 Mar; 186(3):691-700.

[3] Arthritis Care Res. 2016 May;68(5):689-94.

[4] J Bras Pneumol. 2011 Feb; 37(1): 100-109

[5] Arthritis Care Res (Hoboken). 2013 Aug; 65(8): 1307-1315

Disclosure of Interest: None declared

DOI: 10.1136/annrheumdis-2018-eular.7316

\section{AB0757 PERSISTENT CRP ELEVATION IS ASSOCIATED WITH HIGH MORBIDITY IN SYSTEMIC SCLEROSIS}

A. Mitev ${ }^{1}$, D. Feldmann ${ }^{1}$, A.-M. Kanne ${ }^{1}$, T. Hugle ${ }^{2}$, P.M. Villiger ${ }^{3}$, R.E. Voll ${ }^{1}$, S. Finzel ${ }^{1}$, F. Kollert ${ }^{3} .{ }^{1}$ Department of Rheumatology and Clinical Immunology, University Medical Center Freiburg, Freiburg, Germany; ${ }^{2}$ Service de rhumatologie, CHUV, Lausanne; ${ }^{3}$ Rheumatology, Immunology and Allergology, University Hospital Bern Inselspital, Berne, Switzerland

Background: Elevated levels of C-reactive protein (CRP) in systemic sclerosis (SSc) have been linked to early inflammatory stages of the disease.

Objectives: This study has been set to investigate CRP levels in a longitudinal cohort of SSc patients and to correlate these findings to comorbidities and disease characteristics.

Methods: In this retrospective study patients with SSc were recruited from the outpatient clinic of the Department of Rheumatology and Clinical Immunology, University Medical Centre Freiburg. Only patients with at least three consecutive visits at our centre over at least one year were included in this study. CRP serum levels were measured at every visit and categorised as positive if CRP concentrations were $\geq 5 \mathrm{mg} / \mathrm{L}$. Subjects with elevated CRP levels at more than $80 \%$ of visits were defined as being persistently positive. The longitudinal CRP profile was correlated to comorbidities and disease characteristics.

Results: A total of 1852 consecutive visits of 131 SSc patients were analysed. Over the observed time span $\left(91^{136-56}\right.$ months) $19 \%$ ( $\left.n=25\right)$ of patients had continuously elevated CRP levels, whereas in $29 \%(n=38)$ CRP levels were always in the normal range. There was no association between disease duration and CRP levels at first visit $(p>0.5)$. Persistent CRP elevation was associated $(p<0.05)$ with anti-topoisomerase I-antibodies, diffuse cutaneous SSc (dcSSC), modified Rodnan skin score (mRSS), pulmonary fibrosis, and cardiac arrhythmia, whereas no associations with arthritis or malignancy were found $(p>0.1)$. In a patient with dcSSc and persistent elevated CRP even cyclophosphamide and autologous stem cell transplantation did not alter CRP levels.

Conclusions: Persistently elevated CRP levels characterise a more severe fibrotic phenotype and a higher prevalence of cardiac arrhythmias.

Disclosure of Interest: None declared

DOI: 10.1136/annrheumdis-2018-eular.6995

\section{AB0758 \\ EVALUATION OF NAILFOLD VIDEO CAPILLAROSCOPY IN 296 PATIENTS WITH CONNECTIVE TISSUE DISEASES}

G. Kumánovics, G. Nagy, L. Czirják. Rheumatology and Immunology, Universitiy of Pécs, Medical School, Pécs, Hungary

Background: The influence of the presence of Raynaud's phenomenon (RP) on microangiopathy is not well characterised in connective tissue diseases (CTDs). Objectives: To characterise capillary density and capillary morphology by nailfold video capillaroscopy (NVC) in different CTDs with a special focus on the presence/absence of Raynaud's phenomenon (RP) and overlap syndromes.

Methods: 296 patients with systemic sclerosis (SSc), systemic lupus erythematosus (SLE), idiopathic inflammatory myopathies (IIM), Sjogren's syndrome (SS), antiphospholipid syndrome (APS), rheumatoid arthritis (RA), systemic vasculitis and undifferentiated connective tissue disease (UCTD) were investigated by NVC. Control groups consisted of 25 healthy controls $(\mathrm{HC})$ and 22 primary RP (PRP) cases.

Results: The mean capillary density was significantly decreased in SSc, SLE, SS, IIM and APS either compared to HC or PRP cases. Mean microangiopathy evolution score (MES) was higher in SSc, SLE, SS, IIM and APS compared to both $\mathrm{HC}$ and PRP cases. SSc, SLE, SS patients had a significantly higher giant capillary number compared to either $\mathrm{HC}$ or primary RP control cases. Average haemorrhage score was significantly higher in SSc and SS but not in SLE, RA, IIM and APS compared to HC. Average dilatation point was higher in all investigated CTDs compared to HC. Except RA, all investigated CTDs showed significantly higher capillary loss score compared to either PRP controls or HC. RA cases showed such a difference only compared to HC. Average disorganisation score was significantly higher in all CTDs compared to HC but not compared to PRP cases. Ramification score was significantly elevated in SSc and IIM compared to PRP and HC, but no difference was observed in SLE, SS, APS and RA. The mean capillary density was decreased, and MES was higher in RP associated cases compared to patients without RP in cases with SLE, IIM, and RA. Conversely, the presence of RP did not influence these particular parameters in cases with APS and SS. Except SSc, the impact of overlap features was not remarkable on major capillaroscopic findings. SSc capillary pattern was present in $75.3 \%$ of all SSc cases, most commonly SSc late pattern was observed (58.43\%). SSc pattern was present in other CTD cases too $(15.4 \%-40.7 \%)$. SSc patients with a $\mathrm{DLCO}<70 \%$ had significantly more giant capillaries, avascularity, late SSc pattern, increased MES, and lower capillary density compared to cases with DLCO $>70 \%$, but in the other CTDs decreased DLCO was not associated with similar pronounced capillary damage.

Conclusions: Microangiopathy with decreased capillary density and increased MES were present in all CTDs compared to controls. RP caused most pronounced impairment in SLE, IIM and RA compared to non-RP patients, but had no major effect on SS, and APS. Except SSc, the presence of overlap syndrome did not show a remarkable influence on the microvascular abnormalities compared to pure idiopathic cases. SSc late pattern was most frequently observed in CTDs other than SSc, and it may appear even in patients without RP. Previous associations shown by other authors of decreased DLCO and capillary damage in SSC was confirmed. Capillaroscopy is useful not only in IIM and SSc patients but also in all SLE cases and RP associated SS and RA patients. Capillary density and MES are the most valuable parameters.

Disclosure of Interest: None declared DOI: 10.1136/annrheumdis-2018-eular.7435 